\title{
Neurological soft signs in persons with amnestic mild cognitive impairment and the relationships to neuropsychological functions
}

\author{
Hui-jie Li ${ }^{1}$, Peng-yun Wang ${ }^{1,2}$, Yang Jiang ${ }^{3}$, Raymond C K Chan ${ }^{4}$, Hua-li Wang ${ }^{5}$ and Juan Li ${ }^{*}$
}

\begin{abstract}
Background: Neurological abnormalities have been reported in people with amnestic mild cognitive impairment (aMCl). The current study aimed to examine the prevalence of neurological soft signs (NSS) in this clinical group and to examine the relationship of NSS to other neuropsychological performances.

Methods: Twenty-nine people with $\mathrm{aMCl}$ and 28 cognitively healthy elderly people were recruited for the present study. The NSS subscales (motor coordination, sensory integration, and disinhibition) of the Cambridge Neurological Inventory and a set of neuropsychological tests were administered to all the participants.

Results: People with aMCl exhibited significantly more motor coordination signs, disinhibition signs, and total NSS than normal controls. Correlation analysis showed that the motor coordination subscale score and total score of NSS were significantly inversely correlated with the combined Z-score of neuropsychological tests in aMCl group.

Conclusions: These preliminary findings suggested that people with $\mathrm{aMCl}$ demonstrated a higher prevalence of NSS compared to healthy elderly people. Moreover, NSS was found to be inversely correlated with the neuropsychological performances in persons with aMCl. When taken together, these findings suggested that NSS may play a potential important role and serve as a tool to assist in the early detection of aMCl.
\end{abstract}

Keywords: Mild cognitive impairment, Neurological soft signs, Neuropsychological tests

\section{Introduction}

Alzheimer's disease (AD) is the most commonly acquired neurodegenerative disease in elderly people [1]. Mild cognitive impairment (MCI) is considered as a transitional state of normal aging and $\mathrm{AD}$ [2], and was also thought to be the earliest clinical manifestation of common age-related neurological abnormalities, including $\mathrm{AD}[3]$.

Neurological abnormalities have traditionally been divided into "hard signs" and "soft signs" [4]. "Hard signs" usually indicate focal neurological deficits localized within specific brain regions, whereas "soft signs" are conventionally defined as subtle signs without an identifiable or localized brain region [4]. However, most recent studies using brain imaging technologies suggested that

\footnotetext{
* Correspondence: lijuan@psych.ac.cn

${ }^{1}$ Center on Aging Psychology, Key Laboratory of Mental Health, Institute of Psychology, Chinese Academy of Sciences, Beijing, China

Full list of author information is available at the end of the article
}

neurological soft signs (NSS) might be associated with specific brain regions or even brain connections [5-8]. For example, researchers found that higher rates of motor coordination and sensory integration signs were associated with a reduction of grey matter volume of subcortical structures, including putamen, globus pallidus and thalamus in both patients with first-onset schizophrenia and healthy volunteers $[5,6]$. Chan et al. (2006) also showed that brain areas such as bilateral sensorimotor, supplementary motor area, left parietal, and right cerebellum were activated during the fist-edgepalm task [7]. Furthermore, the fist-edge-palm task as a soft sign for motor coordinaiton has been shown to be linked to connectivity between the right inferior and middle prefrontal cortices in healthy control subjects [8].

Patients with AD have also been found to demonstrate significantly higher prevalence of sensory integration and motor coordination signs than healthy controls [9]. Other studies also found NSS abnormalities in patients 
with AD [10,11]. Kumamoto et al. (2000) found elderly people that were cognitively impaired but not demented, had exhibited higher frequency of neurological signs than healthy controls, but lower rate of signs than patients with dementia [12]. Gualtieri et al. (2005) also found that individuals with MCI showed poorer performance in a finger tapping task in which participants were required to tap on the mouse button [13]. A previous study implied that NSS might be a predictor for progression to clinical AD [14]. Yet so far there is no empirical study to systematically explore the NSS in individuals with aMCI. In the present study, we hypothesized that individuals with aMCI would demonstrate more NSS than normal elderly individuals, and that NSS might be taken as an easy-identified neurological marker for early detection of AD.

Neuropsychological performance is considered to be one of the most sensitive and specific markers of prodromal AD [15]. Previous studies indicated that cognitive decline in patients with MCI was associated with widespread structural brain damage [16]. As we know, the executive function aging is the main cause of the cognitive decline in older adults. The so called "frontal hypothesis of cognitive aging" suggests that the prefrontal cortex deteriorated earlier and disproportionately compared to other cortices [17]. In addition, the pathological aging causes declines in volume and microstructural pathology in prefrontal cortex, medial temporal regions, and other regions in grey and white matter density [18-21]. Thus, the motor coordination, sensory integration, and disinhibition subscales of the Cambridge Neurological Inventory (CNI) have been devised to investigate the putative regions of prefrontal lobe, parital lobe, and frontal lobe, respectivelly [22,23]. Studies using sturcutral equaiton modelling showed that there were modest and moderate associations among NSS, executive function, verbal memory, and visual memory in both patients with schizophrenia and healthy controls. Moreover, NSS has been associated with poorer performances in executive function and memory functions in both groups [24]. Recently, Chan et al. (2011) also observed a similar pattern for relationship in healthy older adults [25]. When taken together, these findings suggest that neurolgoical soft signs are capable to capture the similar information measured by conventional neurocognitive tests.

To date, it is still largely unknown about the prevalence rate of NSS in people with MCI and how these signs are related to conventional neuropsychological performances in this clinical group. The purpose of the current study was to explore the prevalence of NSS in aMCI and to examine the relationships of NSS to neuropsychological performances in this clinical group. Given the aforementioned studies, it was hypothesized that
aMCI was associated with a higher prevalence of NSS as compared to healthy older adults. Moreover, NSS was expected to show negative association with neuropsychological performances in this clinical group.

\section{Methods}

\section{Subjects}

Patients with aMCI in this study were recruited from the communities around the Institute of Psychology, Chinese Academy of Sciences and memory clinic of Institute of Mental Health, Peking University. One hundred and sixty-seven participants aged between 6087 years were screened from the residential communities. Among them, 18 were screened as patients with aMCI. Moreover, 11 individuals with aMCI were referred by the psychiatrists of the memory clinic. The diagnoses of aMCI were made according to published criteria $[2,26]$. Participants were interviewed to determine whether they had memory complaint, normal general cognitive function (measured by Mini-Mental State Examination, MMSE) [27], and normal activities of daily living [28]. Objective memory impairment was verified with paired-association learning and portrait characteristics recall tests, which were two subtests of the standardized Clinical Memory Scale [29,30]. For the cut-off scores of these two memory tests, we used a more liberal criterion of 1SD below the norms, as previous studies suggested that the traditional 1.5 SD cut-off would reduce the possibility of detecting early stage memory impairment [31]. An expert team, consisting of psychiatrists and neuropsychologists, made consensus diagnoses on the basis of all available clinical and neuropsychological results.

Healthy controls were also recruited from the same geographic region where people with aMCI were recruited. They were matched for age, gender, and education level with aMCI participants. They were also defined by having normal scores on the pairedassociation learning and portraits characteristics recall [29,30], activities of daily living [28], MMSE [27], and had no complaints of memory problems.

Participants were excluded for both groups if they: (1) had a history of head trauma; (2) had either a disease of the central nervous system or a psychotic disorder; (3) abused alcohol or other substance; or (4) were diagnosed with any form of dementia.

The present study was approved by the ethics committees of the Institute of Psychology, Chinese Academy of Sciences. Written informed consent was obtained from all participants.

\section{Neurological soft signs}

The motor coordination, sensory integration, and disinhibition subscales of the Cambridge Neurological Inventory 
(CNI) was used to assess NSS [22]. The CNI is one of the most commonly used tools to explore the NSS and it has been validated among the Chinese population [32]. The motor coordination subscale includes items assessing rapid motor movements such as finger tapping, finger-thumb opposition, diadockinesia, fist-edge-palm test, and oseretsky test. The sensory integration subscale consists of items evaluating tactile sensation such as extinction test, finger-agnosia, stereognosis, graphesthesia, and left-right orientation. The disinhibition subscale includes items for withholding or inhibiting associated movements. These items include saccade blink and saccade head, wink, go/no-go test, and mirror movements of finger-thumb opposition (left and right hands) and diadocokinesia (left and right hands) [22,23]. The CNI has full instruction for training guidelines, and it also has been shown with good construct and external validity, and interrater reliability [23].

The CNI was administered in a standardized manner according to a fixed order. In the original scale, scoring was made according to standardized anchor points to indicate "normal" response (scored as 0), "equivocal response" (0.5), "abnormal" response (1) or "grossly abnormal" response (2). In the present study, item scores were dichotomized into either "absent" (covering normal or equivocal) or "present" (covering abnormal or grossly abnormal) [24].

\section{Neuropsychological tests}

Participants also received a battery of neuropsychological tests capturing their capabilities in executive function, processing speed, abstract reasoning ability, and memory.

Fluency and Trail-making test (TMT) B were used to assess the executive function. The fluency test includes verbal fluency [33] and writing fluency testing. For verbal fluency, participants were told to speak as many animal names or food names as they could in one minute, respectively. The mean score was considered to be the performance of verbal fluency. For writing fluency, participants were told to write as many Chinese characters as they could for radicals of " $f$ " and “ $\uparrow$ " in a minute, respectively, and the average score was considered to be the performance of writing fluency. For TMT B, 25 circles were printed in black on paper, including the first 13 Arabic numerals and the first 12 Chinese numbers. Participants were asked to connect Arabic numbers and Chinese numbers alternately (for example, 1-一, 2-二, 3-三, etc.), the last link is from the 十二 (12 in Chinese number) to the 13 [34].

Digit span subtest of Wechsler Memory Scale-Revised Chinese version (WMS-RC) was used to assess working memory, and both forward and backward tests were included [35]. Processing speed was evaluated by the digit symbol subtest of Wechsler Adult Intelligence ScaleChinese version (WAIS-C) [36] and TMT A [34]. Similarity subtest of WAIS-C [36] was considered to investigate the verbal abstract reasoning ability, and it was considered to reflect the function of the frontal lobe [37]. Episodic memory was measured by the logic memory, which was selected from WMS-RC [35]. Participants were required to listen to two short stories and then to recall them

Table 1 Demographic variables and neuropsychological tests for aMCl patients and normal older adults

\begin{tabular}{|c|c|c|c|c|c|c|c|c|}
\hline & \multicolumn{3}{|c|}{$\mathrm{aMCl}$} & \multicolumn{3}{|c|}{ Normal Older Adults } & \multirow[t]{2}{*}{$t$} & \multirow[t]{2}{*}{$p$} \\
\hline & $N$ & $M$ & $S D$ & $N$ & $M$ & $S D$ & & \\
\hline Age (years) & 29 & 73.76 & 6.42 & 28 & 71.25 & 6.43 & 1.47 & .15 \\
\hline Education (years) & 29 & 10.97 & 4.82 & 28 & 13.89 & 3.12 & -2.71 & .009 \\
\hline Activities of daily living & 29 & 14.93 & 1.36 & 28 & 14.54 & 1.37 & 1.09 & .28 \\
\hline MMSE & 29 & 26.07 & 2.33 & 28 & 27.61 & 1.95 & -2.70 & .009 \\
\hline Paired-association learning & 29 & 4.45 & 3.06 & 28 & 11.14 & 3.29 & -7.95 & $<.001$ \\
\hline Portraits characteristics recall & 29 & 4.00 & 3.76 & 28 & 9.39 & 5.09 & -4.56 & $<.001$ \\
\hline \multicolumn{9}{|l|}{ Neuropsychological tests } \\
\hline Verbal fluency & 29 & 18.81 & 4.35 & 28 & 22.64 & 4.26 & -3.36 & .001 \\
\hline Writing fluency & 27 & 4.61 & 1.95 & 26 & 5.23 & 1.93 & -1.16 & .25 \\
\hline Digit symbol & 27 & 30.30 & 10.50 & 27 & 35.81 & 7.53 & -2.22 & .031 \\
\hline TMT A (seconds) & 29 & 51.07 & 23.59 & 24 & 44.21 & 16.20 & 1.21 & .23 \\
\hline TMT B (seconds) & 28 & 113.11 & 75.05 & 24 & 78.58 & 35.94 & 2.06 & .045 \\
\hline Digit span-forward & 26 & 7.31 & 1.64 & 27 & 7.85 & 1.32 & -1.33 & .19 \\
\hline Digit span-backward & 26 & 4.15 & 1.32 & 27 & 4.59 & 1.72 & -1.04 & .30 \\
\hline Logic memory & 27 & 5.06 & 2.51 & 27 & 8.44 & 2.89 & -4.60 & $<.001$ \\
\hline Similarity & 29 & 15.41 & 4.20 & 28 & 18.36 & 3.28 & -2.94 & .005 \\
\hline
\end{tabular}


immediately; the memory performance was evaluated with the average score of the two stories.

\section{Statistical analysis}

Statistical analyses were carried out with the Statistical Package for Social Sciences (SPSS) version 13.0. First, Chi-square test was performed to compare the prevalence rate of NSS in patients with aMCI and healthy elderly people. Second, since years of education was significant between two groups, it was controlled as a covariate in analyses. A MANCOVA was used to analyze the group differences in NSS with Bonferroni correction. The effect sizes of the group comparisons were calculated in terms of Cohen's d [38]. Finally, Pearson correlation analysis was used to test the relationships between NSS and neuropsychological performance in patients with aMCI.

\section{Results}

\section{Demographics}

Twenty-nine patients with aMCI (11 males) and 28 normal control participants (15 males) were included in the present study. The Chi square test indicated that there was no significant group differences in gender ratio $\left(\chi^{2}=1.41, p=.29\right)$. As demonstrated in Table 1 , there were no significant group differences in age and activities of daily living. Patients with aMCI received significant lower years of education. They also showed significant lower performance in MMSE, the pairedassociation learning and portrait characteristics recall tests. We also examined the medical histories in the two groups. For aMCI group, 21 out of 29 older adults reported medical histories, among them 14 suffered from hypertension and/or diabetes mellitus and/or coronary heart disease, 3 had cataract or other strabismus, 2 had cavity infarction, and 2 had benign prostate hypertrophy or mammary gland hyperplasia. Nineteen of the 28 healthy older adults reported medial histories. Specially, 14 had hypertension and/or diabetes mellitus and/ or coronary heart disease, 2 had ocular fundus disease or astigmatism, 2 had cervical vertebral disease or stomach disease, and 1 had sleep disorder. The Chi square test indicated that there was no significant group differences in the ratio of suffering from chronic diseases $\left(\chi^{2}=.14\right.$, $p=.78$ ). For the neurocognitive tests, significant group differences were found in verbal fluency, digit symbol, TMT B, logic memory, and similarity, while no significant differences were found in writing fluency, TMT A, forward and backward of digit span.

\section{Comparison of NSS between older adults with $\mathrm{aMCl}$ and normal controls}

The prevalence rate of "present" NSS in aMCI and normal controls are shown in Table 2. The Chi-square test showed that people with aMCI exhibited higher prevalence rate of NSS in most of the items, especially in fistedge-palm in left hand, oseretsky test in motor coordination subscale, and go/no-go in disinhibition subscale.

The MANCOVA results indicated that patients with aMCI demonstrated significantly more dysfunctions in motor coordination $(F(1,54)=6.95, p=.011)$, disinhibition $(F(1,54)=6.78, p=.012)$, and the total NSS $(F(1$, $54)=8.25, p=.006)$. For the sensory integration, there was no significant differences between the two groups ( $F$ $(1,54)=.06, p=.82)$. Figure 1 shows the NSS profiles of patients with aMCI and healthy controls. The effect sizes

Table 2 Prevalence rate of individual items of neurological signs in persons with aMCl $(n=29)$ and normal control $(n=28)$

\begin{tabular}{|c|c|c|c|c|}
\hline NSS items & $\mathrm{aMCl}$ & $\begin{array}{l}\text { Normal } \\
\text { control }\end{array}$ & $x^{2}$ & $p$ \\
\hline \multicolumn{5}{|l|}{ Motor coordination } \\
\hline Left Finger-thumb tapping & $20.7 \%$ & $0 \%$ & 6.48 & .023 \\
\hline Right Finger-thumb tapping & $13.8 \%$ & $3.6 \%$ & 1.86 & .35 \\
\hline Left Finger-thumb opposition & $27.6 \%$ & $21.4 \%$ & .29 & .76 \\
\hline Right Finger-thumb opposition & $17.2 \%$ & $10.7 \%$ & .50 & .71 \\
\hline Left Diadocokinesia & $17.2 \%$ & $10.7 \%$ & .50 & .71 \\
\hline Right Diadocokinesia & $13.8 \%$ & $7.1 \%$ & .67 & .67 \\
\hline Left Fist-edge-palm & $51.7 \%$ & $10.7 \%$ & 11.01 & .001 \\
\hline Right Fist-edge-palm & $27.6 \%$ & $7.1 \%$ & 4.12 & .079 \\
\hline Oseretsky test & $58.6 \%$ & $28.6 \%$ & 5.22 & .033 \\
\hline \multicolumn{5}{|l|}{ Sensory integration } \\
\hline Extinction & $10.3 \%$ & $3.6 \%$ & 1.00 & .61 \\
\hline Left Finger Agnosia & $69.0 \%$ & $42.9 \%$ & 3.94 & .064 \\
\hline Right Finger Agnosia & $34.5 \%$ & $39.3 \%$ & .14 & .79 \\
\hline Left Stereognosia & $0 \%$ & $0 \%$ & NA & NA \\
\hline Right Stereognosia & $0 \%$ & $0 \%$ & NA & NA \\
\hline Left Graphesthesia & $13.8 \%$ & $25.0 \%$ & 1.15 & .33 \\
\hline Right Graphesthesia & $6.9 \%$ & $10.7 \%$ & .26 & .67 \\
\hline Left-Right Orientation & $13.8 \%$ & $14.3 \%$ & .003 & .99 \\
\hline \multicolumn{5}{|l|}{ Disinhibition } \\
\hline $\begin{array}{l}\text { Left Mirror Movement of Finger } \\
\text { Opposition }\end{array}$ & $6.9 \%$ & $0 \%$ & 2.00 & .49 \\
\hline $\begin{array}{l}\text { Right Mirror Movement of Finger } \\
\text { Oppostion }\end{array}$ & $10.3 \%$ & $3.6 \%$ & 1.00 & .61 \\
\hline Left Mirror Movement of Diadocokinesia & $3.4 \%$ & $3.6 \%$ & .001 & .99 \\
\hline Right Mirror Movement of Diadocokinesia & $24.1 \%$ & $7.1 \%$ & 3.10 & .14 \\
\hline Saccade Blink & $17.2 \%$ & $7.1 \%$ & 1.35 & .42 \\
\hline Saccade Head & $27.6 \%$ & $10.7 \%$ & 2.60 & .18 \\
\hline Wink & $10.3 \%$ & $21.4 \%$ & 1.32 & .30 \\
\hline Go No-Go Stimulus & $44.8 \%$ & $10.7 \%$ & 8.21 & .007 \\
\hline
\end{tabular}

p-values: two sided, Fisher's Exact Test. 


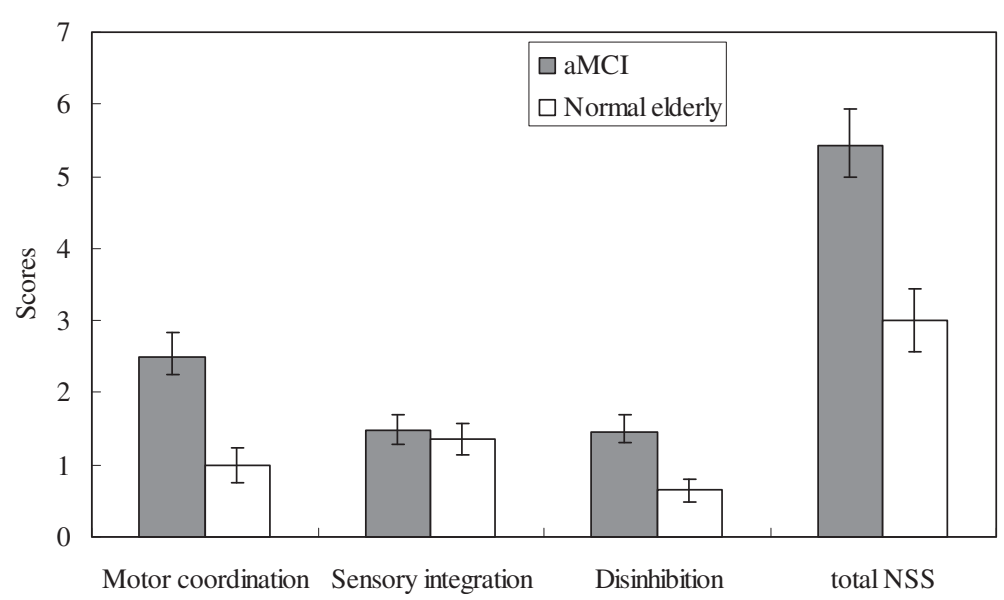

Figure 1 Comparisons of neurological soft signs in persons with aMCl and normal elderly. Error bars represent $95 \%$ confidence intervals.

for motor coordination (Cohen's $\mathrm{d}=0.93$ ) and total NSS score (Cohen's $\mathrm{d}=0.96)$ reached large, while the effect sizes were moderate for disinhibition (Cohen's $d=0.76$ ) and negligible for the sensory integration (Cohen's $\mathrm{d}=0.12)$.

\section{Correlations between NSS and neuropsychological tests in patients with aMCI}

The neuropsychological performance was indexed by the combined Z-score of the verbal fluency and writing fluency, digit symbol, forward and backward of digit span, TMT A and B, similarities, and logic memory.

Pearson correlation analysis showed that the total NSS score and motor coordination subscale were significantly negatively correlated with combined Z-score of neuropsychological tests $(r=-.53, p<.01 ; r=-.42, p<.05)$, the sensory integration and disinhibition subscales were not found to be significantly correlated with combined Zscore of neuropsychological tests $(r=-.19, p>.05$; $r=-.33, p>.05)$.

\section{Discussion}

Our results revealed that patients with aMCI exhibited a higher prevalence rate of neurological abnormalities than normal control participants. The preliminary results also suggested NSS and neuropsychological tests might reflect somewhat similar information for the brain functioning. The present study indicated that NSS may play an important role and serve as a tool to assist in the early detection of aMCI.

To our knowledge, this is the first study to investigate the NSS in older adults with aMCI. Our results demonstrated that aMCI individuals displayed significantly more neurological abnormalities than normal controls on motor coordination, disinhibition, and the total NSS. The effect sizes of group comparisons were large for motor coordination and total score and moderate for disinhibition. The findings were similar to previous studies focused on normal elderly people and people with pathological aging disease. With the same scale, Chan et al. (2011) found that NSS was common among elderly people, and the prevalence rate of soft signs increased with advancing age [25]. In a neurological examination including few soft signs (etc., saccadic eye movement), the older adults who were with cognitively impaired but without dementia were found to produce a higher prevalence of signs than the normal controls [12]. Patients with $\mathrm{AD}$ and other several forms of dementia were also found to have higher prevalence of NSS than those without dementia [9].

The current study indicated that older adults with aMCI showed more motor dysfunctions than cognitively normal older people. Previous findings indicated that people with MCI and mild AD demonstrated dysfunctions in equilibrium and limb coordination [39]. Other studies confirmed that people with aMCI performed worse on tasks involving fine and complex motor functions than normal older adults [40,41]. Lam et al. (2005) also found motor coordination signs were very sensitive in discriminating patients with or without dementia [9]. Signs such as primitive reflexes and mirror movements were classified as disinhibition, which included the signs of spurious movements in a time and place where it was not expected to occur [22]. In the present study, patients with aMCI demonstrated significantly more signs than normal controls in the disinhibition subscale. Similar findings were also reported in previous studies. Franssen et al. (1991) found participants in an early stage of AD showed higher mean score of deep tendon reflexes than normal elderly people, while patients with a later stage of $\mathrm{AD}$ demonstrated significantly increased prevalence of sucking reflexes compared with normal older adults 
and patients with the early stage of $\mathrm{AD}$ [10]. Furthermore, more primitive reflexes were found in the more terminal stages of AD [11]. Previous studies also found that MCI patients showed inhibition impairments in some neuropsychological tasks, such as go/no-go task [42], Stroop task [43,44], Hayling test [45], and Flanker test [46].

The correlations between NSS and neuropsychological functions have significant implications. Our results indicated that the total NSS score was negatively correlated with the combined Z-score of neuropsychological tests in aMCI group. The current results were consistent with our recent findings in normal older adults that NSS had moderate associations with neurocognition function [25]. The present study provided further evidence that the two measures were more or less statistically equivalent to capture the similar brain functions. These results support in part the assumption that motor coordination might be an indicator of the prefrontal lobe function $[7,8]$. However, the correlation analysis also indicated that sensory integration and disinhibition did not have significant correlations with the neuropsychological tests. The reason might be as mentioned previously, sensory integration and disinhibition subscales were considered to reflect parietal and frontal lobe functions respectively [22,23], and aMCI patients showed relatively less impairment in these two subscales. Whereas, there were no sensitive neuropsychological tasks to reflect frontal lobe function and no tasks specialized to measure the parietal lobe function in current study. To better understand whether there are same neural substrates between NSS and neuropsychological functions, future studies with larger samples and more comprehensive neuropsychological tasks are needed.

The present study has several limitations. First, the sample size of the study is relatively small. More studies with larger sample sizes are needed in order to confirm the neurological dysfunctions in persons with aMCI, which could help to further clarify the early neurological abnormalities of aMCI. Second, some of the aMCI participants were from a memory clinic, and these participants were not scored by blinded raters, which might bring some bias during assessment. Future studies should overcome the difficulties and assess the NSS with blind raters. Third, aMCI participants were recruited from both community and the memory disorder clinic, while the health elderly were only selected from the community residents. This may lead to differential selection bias. Fourth, due to the mean age of participants in both groups were older than 70 years, 40 out of the 57 participants suffered from one or more chronic diseases such as hypertension, diabetes mellitus, coronary heart disease and other diseases. Also, unfortunately quite a number of the elderly could not recall the exact type and dose of medicines they have been taking during the assessment and we did not further follow up with these; therefore, we are uncertain whether their medication will influence their neuropsychological or NSS performances. Moreover, the present study adopted the traditional diagnosis criteria of aMCI proposed by Petersen and colleagues $[2,26]$, which was called core clinical criteria by the National Institute on Aging and Alzheimer's Association workgroup [47]. The workgroup also recommended new diagnosis criteria: research criteria, which incorporated the biomarker based on imaging or cerebrospinal fluid measures into the core clinical criteria [47]. Studies adopting more stringent research criteria for screening aMCI are needed to confirm the current findings.

Our current findings have shown that aMCI patients demonstrated significantly higher prevalence of NSS than healthy older adults. The total scores of NSS were significantly correlated with the combined Z-score of neuropsychological tests in aMCI group. NSS has been found to be indicative of the cognitive decline and brain dysfunction $[4,23]$. The observed impairment of NSS in aMCI contributes further evidence to the literature on neurological deficits in pathological aging diseases. Given the assessment is simple, non-invasive and timesaving, neurological soft sign test may be used as an assistant tool for the bedside clinical examination of mild cognitive impairment.

\section{Abbreviations}

AD: Alzheimer's disease; aMCl: Amnestic mild cognitive impairment; CNI: Cambridge Neurological Inventory; MCl: Mild cognitive impairment; MMSE: Mini Mental State Examination; NSS: Neurological soft signs; TMT: Trail-making test; WMS-RC: Wechsler Memory Scale-Revised Chinese version; WAIS-C: Wechsler Adult Intelligence Scale-Chinese version.

\section{Competing interests}

The authors declare that they have no competing interest.

\section{Authors' contributions}

HJL designed the study, analyzed the data, and wrote up the first draft of the paper. JL conceived the idea and participated in the design of the study and the writing up of the paper. PYW collected the data and assisted data analysis. RCKC and $Y J$ contributed to the writing up of the manuscript, and HLW helped aMCl participants' recruitment and diagnosis. All authors read and approved the final manuscript.

\section{Acknowledgements}

We thank Ting Zhou, Bing Li, and Xin Li for their contribution of data collection and McKinley Heflin's assistance for English editing. This study was supported by National Natural Science Foundation of China (30770725, 31000465, 30911120494), National Science \& Technology Pillar Program of China (2009BAl77B03), and Knowledge Innovation Project of the Chinese Academy of Sciences (KSCX2-YW-R-256 \& KSCX2-EW-J-8), and a grant from the National Outstanding Young Investigator Award from National Science Foundation of China (81088001).

\section{Author details}

${ }^{1}$ Center on Aging Psychology, Key Laboratory of Mental Health, Institute of Psychology, Chinese Academy of Sciences, Beijing, China. ${ }^{2}$ Graduate School, Chinese Academy of Sciences, Beijing, China. ${ }^{3}$ Department of Behavioral Science, College of Medicine, University of Kentucky, Kentucky, USA. 
${ }^{4}$ Neuropsychology and Applied Cognitive Neuroscience Laboratory, Key Laboratory of Mental Health, Institute of Psychology, Chinese Academy of Sciences, Beijing, China. ${ }^{5}$ Department of Geriatric Psychiatry, Institute of Mental Health, Peking University, Beijing, China.

Received: 14 November 2011 Accepted: 28 May 2012 Published: 7 June 2012

\section{References}

1. Touchon J, Ritchie K: Prodromal cognitive disorder in Alzheimer's disease. Int J Geriatr Psychiatry 1999, 14:556-563.

2. Petersen RC, Doody R, Kurz A, Mohs RC, Morris JC, Rabins PV, Ritchie K, Rossor M, Thal L, Winblad B: Current concepts in mild cognitive impairment. Arch Neurol 2001, 58:1985-1992.

3. Bennett DA, Schneider JA, Bienias JL, Evans DA, Wilson RS: Mild cognitive impairment is related to Alzheimer disease pathology and cerebral infarctions. Neurology 2005, 64:834-841.

4. Mohr F, Hubmann W, Albus M, Franz U, Hecht S, Scherer J, Binder J, Sobizack N: Neurological soft signs and neuropsychological performance in patients with first episode schizophrenia. Psychiatry Res 2003, 121:21-30.

5. Dazzan P, Morgan KD, Orr KG, Hutchinson G, Chitnis X, Suckling J, Fearon P, Salvo J, McGuire PK, Mallett RM, et al: The structural brain correlates of neurological soft signs in AESOP first-episode psychoses study. Brain 2004, 127:143-153.

6. Dazzan P, Morgan KD, Chitnis X, Suckling J, Morgan C, Fearon P, McGuire $P K$, Jones PB, Leff J, Murray RM: The structural brain correlates of neurological soft signs in healthy individuals. Cereb Cortex 2006, 16:1225-1231

7. Chan RCK, Rao H, Chen EE, Ye B, Zhang C: The neural basis of motor sequencing: an fMRI study of healthy subjects. Neurosci Lett 2006, 398:189-194.

8. Rao H, Di X, Chan RCK, Ding Y, Ye B, Gao D: A regulation role of the prefrontal cortex in the fist-edge-palm task: evidence from functional connectivity analysis. Neuroimage 2008, 41:1345-1351.

9. Lam LL, Lui VWC, Chiu HFK: Associations between neurological soft Signs and clinical progression Alzheimer's disease. The Hong Kong Journal of Psychiatry 2005, 15:43-49

10. Franssen EH, Reisberg B, Kluger A, Sinaiko E, Boja C: Cognition-independent neurologic symptoms in normal aging and probable Alzheimer's disease. Arch Neurol 1991, 48:148-154.

11. Benesch CG, McDaniel KD, Cox C, Hamill RW: End-stage Alzheimer's disease. Glasgow Coma Scale and the neurologic examination. Arch Neurol 1993, 50:1309-1315.

12. Kumamoto T, Sannomiya K, Ueyama H, Aoki K, Nakashima T, Nakamura R, Tsuda T: Neurological abnormalities in cognitively impaired but not demented elderly. Acta Neurol Scand 2000, 102:292-298.

13. Gualtieri $C T$, Johnson LG: Neurocognitive testing supports a broader concept of mild cognitive impairment. Am J Alzheimers Dis Other Demen 2005, 20:359-366.

14. Wilson RS, Schneider JA, Bienias JL, Evans DA, Bennett DA: Parkinsonianlike signs and risk of incident Alzheimer disease in older persons. Arch Neurol 2003, 60:539-544.

15. Sarazin M, Berr C, De Rotrou J, Fabrigoule C, Pasquier F, Legrain S, Michel B, Puel M, Volteau M, Touchon J, et al: Amnestic syndrome of the medial temporal type identifies prodromal AD: a longitudinal study. Neurology 2007, 69:1859-1867.

16. Van Der Flier WM, Van Den Heuvel DM, Weverling-Rijnsburger AW, Spilt A, Bollen EL, Westendorp RG, Middelkoop HA, Van Buchem MA: Cognitive decline in $A D$ and mild cognitive impairment is associated with global brain damage. Neurology 2002, 59:874-879.

17. West RL: An application of prefrontal cortex function theory to cognitive aging. Psychol Bull 1996, 120:272-292.

18. Apostolova LG, Steiner CA, Akopyan GG, Dutton RA, Hayashi KM, Toga AW, Cummings JL, Thompson PM: Three-dimensional gray matter atrophy mapping in mild cognitive impairment and mild Alzheimer disease. Arch Neurol 2007, 64:1489-1495.

19. Chen TF, Chen YF, Cheng TW, Hua MS, Liu HM, Chiu MJ: Executive dysfunction and periventricular diffusion tensor changes in amnesic mild cognitive impairment and early Alzheimer's disease. Hum Brain Mapp 2009, 30:3826-3836.
20. Chua TC, Wen W, Chen XH, Kochan N, Slavin MJ, Trollor JN, Brodaty H, Sachdev PS: Diffusion Tensor Imaging of the Posterior Cingulate is a Useful Biomarker of Mild Cognitive Impairment. Am J Geriatr Psychiatry 2009, 17:602-613.

21. Devanand DP, Pradhaban G, Liu X, Khandji A, De Santi S, Segal S, Rusinek H, Pelton GH, Honig LS, Mayeux R, et al: Hippocampal and entorhinal atrophy in mild cognitive impairment - Prediction of Alzheimer disease. Neurology 2007, 68:828-836.

22. Chen EY, Shapleske J, Luque R, McKenna PJ, Hodges JR, Calloway SP, Hymas NF, Dening TR, Berrios GE: The Cambridge Neurological Inventory: a clinical instrument for assessment of soft neurological signs in psychiatric patients. Psychiatry Res 1995, 56:183-204.

23. Chan RCK, Gottesman II: Neurological soft signs as candidate endophenotypes for schizophrenia: A shooting star or a Northern star? Neurosci Biobehav Rev 2008, 32:957-971.

24. Chan RCK, Wang Y, Wang L, Chen EYH, Manschreck TC, Li ZJ, Yu X, Gong QY: Neurological Soft Signs and Their Relationships to Neurocognitive Functions: A Re-Visit with the Structural Equation Modeling Design. PLoS One 2009, 4:e8469.

25. Chan RCK, Xu T, Li HJ, Zhao Q, Liu HH, Wang Y, Yan C, Cao XY, Wang YN, Shi YF, Dazzan P: Neurological abnormalities and neurocognitive functions in healthy elder people: a structural equation modeling analysis. Behav Brain Funct 2011, 7:32.

26. Petersen RC: Mild cognitive impairment as a diagnostic entity. J Intern Med 2004, 256:183-194.

27. Wang ZY, Zhang MY, Qu GY, Chen JX, Zhao J: The application of the Chinese version of Mini Mental State Examination. Shanghai Psychiatry Medicine 1989, 7:108-111 (in Chinese).

28. Yang $C Z$, Tian $J Z$, Zhong J: The blood fat changes in mild cognitive impairment and Alzheimer's disease. Chinese Journal of Gerontology 2007 6:545-548. in Chinese.

29. Clinical Memory Scale Cooperative: Construction of the Clinical Memory Test. Acta Psychologica Sinica 1986, 18:100-108. in Chinese.

30. Clinical Memory Scale Cooperative: The Manual of Clinical Memory Test. revised edth edition. Beijing: Institute of Psychology, Chinese Academy of Sciences; 1996. in Chinese.

31. Crowell T, Luis C, Vanderploeg R, Schinka J, Mullan M: Memory patterns and executive functioning in mild cognitive impairment and Alzheimer's disease. Aging Neuropsychol C 2002, 9:288-297.

32. Chen EYH, Chan RCK: The Cambridge Neurological Inventory: Clinical, demographic, and ethnic correlates. Psychiatric Annals 2003, 33:202-210.

33. Spreen O, Strauss E: A Compendium of Neuropsychological Tests: Administration, Norms, and Commentary. 2nd edition. New York: Oxford University Press; 1998.

34. Zhang YM, Han BX, Verhaeghen P, Nilsson LG: Executive functioning in older adults with mild cognitive impairment: $\mathrm{MCl}$ has effects on planning, but not on inhibition. Aging Neuropsychol C 2007, 14:557-570.

35. Gong YX, Jiang DW, Deng JL, Dai ZS, Zhou QZ, Xie GY, Li Y, Hua XX: Manual of Wechsler Memory Scale: Chinese version. Changsha: Hunan Medical College; 1989

36. Gong YX: Manual of Wechsler Adult Intelligence Scale-Chinese version. Changsha: Chinese Map Press; 1992.

37. Alexander MP, Benson DF, Stuss DT: Frontal lobes and language. Brain Lang 1989, 37:656-691

38. Cohen J: Statistical Power Analysis for the Behavioral Sciences. 2nd edition Hillsdale, NJ: Lawrence Earlbaum; 1988.

39. Franssen EH, Souren LEM, Torossian $\mathrm{CL}$, Reisberg B: Equilibrium and limb coordination in mild cognitive impairment and mild Alzheimer's disease. J Am Geriatr Soc 1999, 47:463-469.

40. Aggarwal NT, Wilson RS, Beck TL, Bienias JL, Bennett DA: Motor dysfunction in mild cognitive impairment and the risk of incident Alzheimer disease. Arch Neurol 2006, 63:1763-1769.

41. Economou A, Papageorgiou SG, Karageorgiou C, Vassilopoulos D: Nonepisodic memory deficits in amnestic MCl. Cogn Behav Neurol 2007, 20:99-106.

42. Hanyu H, Sato T, Takasaki A, Akai T, Iwamoto T: Frontal lobe dysfunctions in subjects with mild cognitive impairment. J Neurol 2009, 256:1570-1571.

43. Bélanger $\mathrm{S}$, Belleville $\mathrm{S}$, Gauthier S: Inhibition impairments in Alzheimer's disease, mild cognitive impairment and healthy aging: Effect of congruency proportion in a Stroop task. Neuropsychologia 2010, 48:581-590. 
44. Traykov L, Raoux N, Latour F, Gallo L, Hanon O, Baudic S, Bayle C, Wenisch E, Remy P, Rigaud AS: Executive functions deficit in mild cognitive impairment. Cogn Behav Neurol 2007, 20:219-224.

45. Brandt J, Aretouli E, Neijstrom E, Samek J, Manning K, Albert MS, Bandeen-Roche K: Selectivity of Executive Function Deficits in Mild Cognitive Impairment. Neuropsychology 2009, 23:607-618.

46. Wylie SA, Ridderinkhof KR, Eckerle MK, Manning CA: Inefficient response inhibition in individuals with mild cognitive impairment.

Neuropsychologia 2007, 45:1408-1419.

47. Albert MS, DeKosky ST, Dickson D, Dubois B, Feldman HH, Fox NC, Gamst A, Holtzman DM, Jagust WJ, Petersen RC: The diagnosis of mild cognitive impairment due to Alzheimer's disease: Recommendations from the National Institute on Aging and Alzheimer's Association workgroup. Alzheimers Dement 2011, 7:270-279.

doi:10.1186/1744-9081-8-29

Cite this article as: Li et al.: Neurological soft signs in persons with amnestic mild cognitive impairment and the relationships to neuropsychological functions. Behavioral and Brain Functions 2012 8:29.

\section{Submit your next manuscript to BioMed Central and take full advantage of:}

- Convenient online submission

- Thorough peer review

- No space constraints or color figure charges

- Immediate publication on acceptance

- Inclusion in PubMed, CAS, Scopus and Google Scholar

- Research which is freely available for redistribution 